\title{
Perfil epidemiológico dos casos notificados de sífilis gestacional e congênita em Curitiba/PR (2014-2019)
}

\section{Epidemiological profile of reported cases of gestational and congenital syphilis in Curitiba/PR (2014-2019)}

Eduardo Massaro Yamashita1', Giórgia Souza Franco², Wellidha Bianca Rocha Amado³, Isabella Kohatsu Arakaki ${ }^{4}$, Daiane Cristina Pazin ${ }^{5}$

1. ORCID: https://orcid.org/0000-0002-3339-9148. Discente do curso de medicina da Universidade Positivo. Universidade Positivo, Curitiba, Paraná, Brasil.

E-mail: eduardoyamashita@terra.com.br

2. ORCID: https://orcid.org/0000-0001-5559-8458. Discente do curso de medicina da Universidade Positivo. Universidade Positivo, Curitiba, Paraná, Brasil.

E-mail: gisofran@hotmail.com

3. ORCID: https://orcid.org/0000-0002-3339-9148. Discente do curso de medicina da Universidade Positivo. Universidade Positivo, Curitiba, Paraná, Brasil.

E-mail:wellidha@hotmail.com

4. ORCID: https://orcid.org/0000-0002-9327-8057. Discente do curso de medicina da Universidade Positivo. Universidade Positivo, Curitiba, Paraná, Brasil.

E-mail: isabellaarakaki@hotmail.com

5. ORCID: https://orcid.org/0000-0002-6626-9147. Médica. Mestre em Ciências da Saúde e docente na Escola de Medicina da Universidade Positivo. Universidade Positivo, Curitiba, Paraná, Brasil.

E-mail: daiane.pazin@up.edu.br.

CONTATO: Autor correspondente: Eduardo Massaro Yamashita | Endereço: Prof. Pedro Viriato Parigot de Souza, 5300 - Cidade Industrial, Curitiba - Paraná Telefone: (41) 987381138 E-mail: eduardoyamashita@terra.com.br

\section{RESUMO}

A sífilis é um problema de saúde pública no Brasil, que está relacionada a altos índices de morbimortalidade materna e perinatal. $\mathrm{O}$ estudo em questão 
busca descrever o perfil epidemiológico dos casos notificados de sífilis gestacional e congênita em Curitiba, Paraná, no período de 2014 a 2019. Foram notificados 2.599 casos de sífilis gestacional e 891 casos de sífilis congênita. $79,8 \%$ das gestantes eram brancas, $48,9 \%$ dos diagnósticos de sífilis gestacional firmaram-se no primeiro trimestre de gestação, e $88,9 \%$ dos casos eram de sífilis latente. Com relação a sífilis congênita, $83,3 \%$ das gestantes realizaram o pré-natal e $74,3 \%$ foram diagnosticadas durante o prénatal e $63,7 \%$ das gestantes realizaram o tratamento inadequado. Portanto, os casos sífilis gestacional e congênita foram notificados em sua maioria no primeiro trimestre de gestação, em estágio de sífilis latente e em gestantes que realizaram o pré-natal e tratamento inadequado para sífilis.

DESCRITORES: Gestantes. Sífilis Congênita. Sífilis. Epidemiologia Descritiva

\section{ABSTRACT}

Syphilis is a public health problem in Brazil, which is related to high rates of maternal and perinatal morbidity and mortality. This study describes the epidemiological profile of reported cases of gestational and congenital syphilis in Curitiba, Paraná, from 2014 to 2019. In our study, 2,599 cases of gestational syphilis and 891 cases of congenital syphilis were reported. $79.8 \%$ of pregnant women were white, $48.9 \%$ two diagnoses of gestational syphilis in the first trimester of pregnancy, and $88.9 \%$, two cases were of latent syphilis. Regarding congenital syphilis, $83.3 \%$ of pregnant women received or received prenatal care and $74.3 \%$ were diagnosed during or prenatal and $63.7 \%$ of pregnant women received or received inadequate treatment. Therefore, gestational and congenital syphilis cases were mostly reported in the first trimester of pregnancy, in latent syphilis stage and in pregnant women who underwent prenatal care and inadequate treatment for syphilis.

DESCRIPTORS: Pregnant Women. Syphilis, Congenital. Syphilis. Epidemiology, Descriptive 


\section{INTRODUÇÃO}

sífilis é uma doença infectocontagiosa notadamente estabelecida como um $\Delta$

problema de saúde pública mundial, pois mesmo com a utilização da penicilina a partir de 1940 e com a melhoria nos cuidados à saúde, a Organização Mundial de Saúde (OMS) estima que 5,6 milhões de novos casos de sífilis ocorram entre adolescentes e adultos com idades entre 15 e 49 anos $^{1,2}$. No Brasil, segundo o Ministério da Saúde, em 2018 foram notificados 159.131 casos de sífilis adquirida, 62.599 casos de sífilis em gestantes e 26.219 casos de sífilis congênita, com um aumento de $5,2 \%$ nas notificações em relação a $2017^{3}$.

A doença está diretamente relacionada a elevação dos índices de morbimortalidade materna e perinatal ${ }^{4}$, e quando não tratada adequadamente (início em até 30 dias antes do parto e tratamento adequado para o estágio clínico da sífilis com penicilina benzatina), implica em desfechos clínicos desfavoráveis ${ }^{5}$. Dessa forma, todos os casos devem ser notificados ao Sistema de Informação de Agravos de Notificação (SINAN), permitindo assim, o controle numérico e a vigilância sobre a doença ${ }^{3}$. Apesar disso, acredita-se haver uma subnotificação dos casos em algumas regiões devido ao sub-registro de intercorrências, resultados de exames, tratamentos realizados e fatores de risco ${ }^{5,6}$.

Sabe-se que a sífilis gestacional sem tratamento adequado pode gerar consequências como abortamento e nascimento prematuro ${ }^{5}$. Dessa forma, o acompanhamento pré-natal adequado é a melhor forma de prevenir a ocorrência da sífilis congênita, por isso é preconizado na atenção básica de Curitiba, Paraná, a realização de rastreamento da sífilis por quimioluminescência para todas as gestantes nos três trimestres da gestação?

É também necessário o acompanhamento sorológico durante o tratamento para confirmar a efetividade da terapêutica ${ }^{2}$ e evitar as consequências da doença não tratada, que incluem aborto, prematuridade e óbito fetal ou neonatal, além de comprometimento oftalmológico, auditivo e neurológico na criança ${ }^{5}$. Pode-se afirmar que a doença tem um impacto grave e inestimável na saúde da gestante e do feto, mas é evitável quando o serviço de saúde atende às necessidades da paciente ${ }^{5}$. Da mesma forma, a disseminação de dados e conhecimento sobre a epidemiologia da 
doença pode auxiliar no delineamento de formas de prevenção e redução da transmissão vertical ${ }^{5}$. Assim, este estudo tem como objetivo descrever os indicadores epidemiológicos relativos aos casos notificados de sífilis congênita e gestacional na cidade de Curitiba, Paraná, Brasil, no período entre 2014 e 2019.

\section{MÉTODO}

Estudo epidemiológico, transversal e descritivo que utilizou a base de dados públicos secundários do Departamento de Informática do SUS (DATASUS) por meio do Sistema de Informações sobre Nascidos Vivos (Sinasc) e SINAN ${ }^{8}$ (sem as variáveis de identificação), que possui um formulário padronizado com informações sociodemográficas e clínicas preenchidas por profissionais de saúde pela ficha de notificação compulsória, sendo Curitiba, a capital do Paraná, a cidade para análise dos dados. Um município com população estimada de 1.948.626 habitantes em 2020 e com um Índice de Desenvolvimento Humano Municipal (IDHM) de 0,8239.

Foram incluídos no estudo pacientes notificados com sífilis gestacional e congênita residentes em Curitiba no período de 2014 a 2019 que atenderam os critérios diagnósticos segundo o Ministério da Saúde ${ }^{9}$. Foram excluídos os casos duplicados identificados pelo SINAN, ou seja, aqueles em duplicidade de registros de agravos notificados como casos suspeitos e/ou confirmados, quando o mesmo paciente foi notificado mais de uma vez pela mesma ou outra unidade de saúde, para o mesmo agravo, durante o período prodrômico de um mesmo evento.

As variáveis analisadas foram selecionadas por meio de informações presentes no SINAN ${ }^{8}$ referente a sífilis gestacional e congênita:

1. Sociodemográficas maternas na Sífilis Gestacional e Congênita:

- Faixa etária (em anos 10 a 14; 15 a 19; 20 a 29; 30 a 39; 40 ou mais; Ignorada)

- Escolaridade (Analfabeto; $1^{\underline{a}}$ a $4^{a}$ série incompleta; $4^{a}$ série completa; $5^{\underline{a}}$ a $8^{\underline{a}}$ série incompleta; Ensino fundamental completo; Médio incompleto; Médio completo; Superior incompleto; Superior completo; Ignorado)

- Raça ou Cor (Preta; Parda; Indígena; Branca; Amarela; Ignorada)

2. Variáveis da Sífilis Gestacional:

- Momento do diagnóstico (trimestre de gestação $1^{\circ}$ - trimestre; $2^{\circ}$ trimestre; $3^{\circ}$ trimestre; Idade gestacional ignorada)

- Esquema de tratamento (Penicilina; outro esquema; não realizado; Ignorado)

- Classificação clínica no momento do diagnóstico (Sífilis primária; Sífilis secundária; Sífilis terciária; Sífilis latente; ignorado)

3. Variáveis da Sífilis Congênita: 
- Idade de diagnóstico de Sífilis congênita (Menos de 7 dias; 7 a 27 dias; 28 a 364 dias; 1 ano; 2 a 4 anos; 5 a 12 anos; ignorado)

- Classificação clínica no momento do diagnóstico (Sífilis congênita recente, Sífilis congênita tardia; Aborto por Sífilis; Natimorto por Sífilis)

- Realização de pré-natal materna (Sim; não; ignorado)

- Momento do diagnóstico da sífilis materna (Durante o pré-natal; no momento do parto/curetagem; após o parto; não realizado; Ignorado)

- Esquema de tratamento da mãe (Adequado; Inadequado; não realizado; Ignorado)

- Tratamento do parceiro da mãe (Sim; Não; Ignorado)

Para o cálculo do coeficiente de incidência da sífilis congênita, utilizou-se o número de casos novos por ano, dividido pelo número de nascidos vivos do mesmo ano e multiplicado por 1.000 e para a análise do coeficiente de prevalência de sífilis gestacional utilizou-se o número de casos notificados por ano, dividido pelo número de nascidos vivos do mesmo ano e multiplicado por $1.000^{11}$. As informações foram exportadas do SINAN e posteriormente tabuladas no programa TabWin ${ }^{\circledR}$, sendo também utilizados recursos dos programas Epi Info® e Microsoft Office Excel® para a realização das tabelas e estatísticas.

O estudo foi aprovado pelo Comitê de Ética em Pesquisa do Centro de Estudos Superiores Positivo LTDA, sob o parecer nํ․ 4.260.971, em 05 de setembro de 2020.

\section{RESULTADOS}

Foram identificados 2.599 casos de sífilis gestacional e 891 casos de sífilis congênita no período de 2014 a 2019. Em 2019 (21,9/1.000 nascidos vivos) foi identificado o maior coeficiente de prevalência da sífilis gestacional, o que representou um aumento de 69,53\% quando comparado ao mesmo coeficiente em 2014 (12,8/1.000 nascidos vivos). Em 2017 (8,3/1.000 nascidos vivos) foi evidenciado o maior coeficiente de incidência da sífilis congênita, representando um aumento de 50,9\% quando comparado a taxa de incidência da sífilis congênita em 2014 (5,5/1.000 nascidos vivos) (Figura 1). 
Figura 1. Coeficiente de incidência de sífilis congênita e de prevalência de sífilis gestacional (por 1.000 nascidos vivos/ano) no município de Curitiba, Paraná, 20142019.

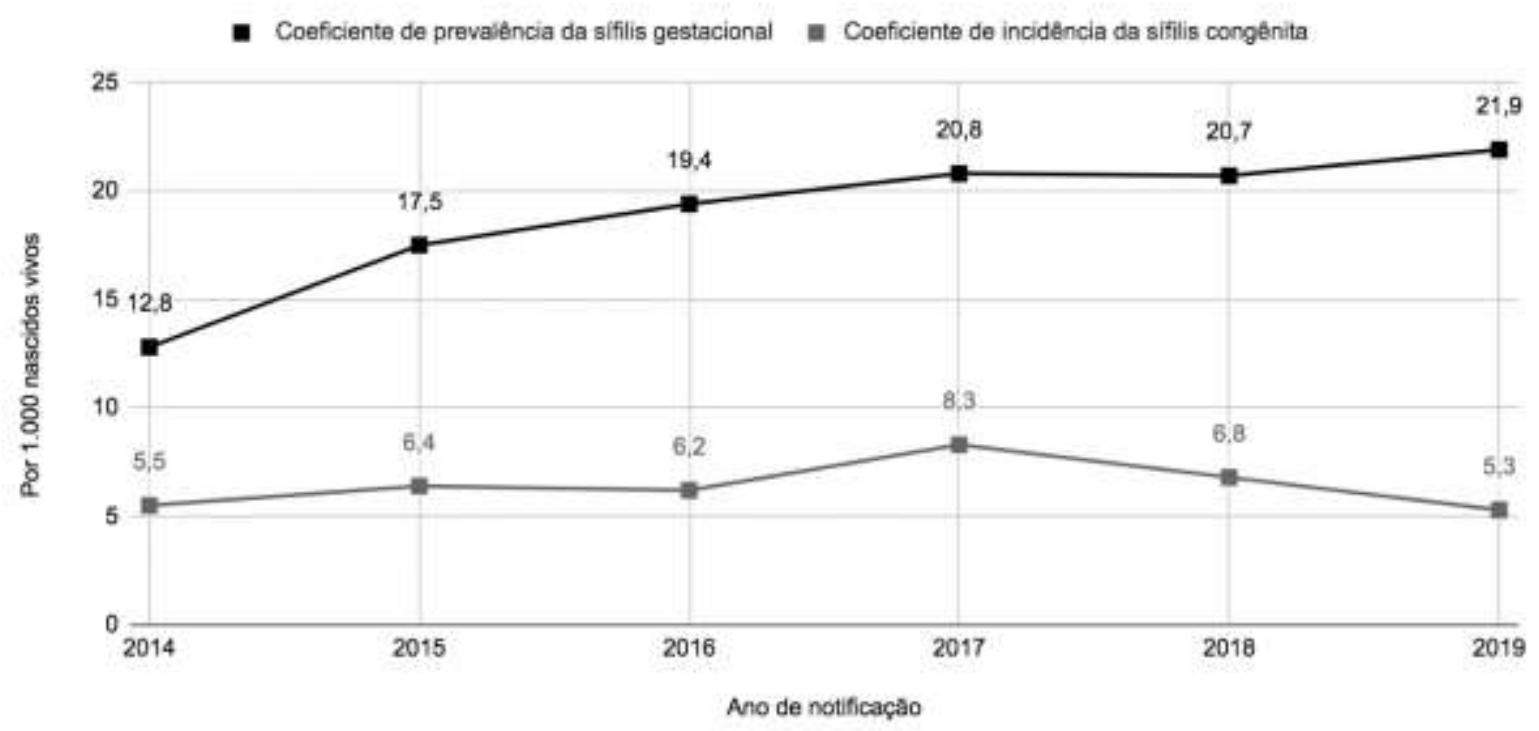

Fonte: Elaborado pelos autores (2021)

Em 2017 houve 474 casos de sífilis gestacional, representando a maioria dos casos de sífilis em gestante com 18,23\%. 55,1\% das grávidas diagnosticadas com a doença encontravam-se na faixa etária de 20-29 anos. Mais de 2/3 eram brancas (79,8\%), 22,2\% tinham escolaridade ignorada, e $21,8 \%$ com $5^{\text {a }}$ a $8^{\text {a }}$ série incompleta. $48,9 \%$ tiveram o diagnóstico no primeiro trimestre da gestação, ao passo que o diagnóstico no terceiro trimestre foi o menos frequente com 21,9\%. Em relação a classificação clínica no momento do diagnóstico, a sífilis latente representou 88,9\% dos casos, seguidos pela sífilis primária $(5,2 \%)$, sífilis terciária $(1,9 \%)$ e sífilis secundária $(0,8 \%)$. O esquema de tratamento mais utilizado foi com a penicilina $(90,8 \%)$, mas $8,6 \%$ das gestantes não realizaram tratamento (Tabela 1) e (Tabela 2).

Tabela 1. Características sociodemográficas e epidemiológicas das gestantes com sífilis ( $\mathrm{n}=2.599)$, notificadas no SINAN no município de Curitiba, Paraná, 2014-2019.

\begin{tabular}{lrr}
\hline Variáveis da sífilis gestacional & $\mathbf{n}$ & $\%$ \\
\hline Faixa etária da gestante (em anos) & & \\
10 a 14 & 14 & 0,5 \\
15 a 19 & 507 & 19,5 \\
20 a 29 & 1.432 & 55,1 \\
30 a 39 & 556 & 21,4 \\
40 ou mais & 90 & 3,5
\end{tabular}




\section{Momento do diagnóstico (trimestre de gestação)}

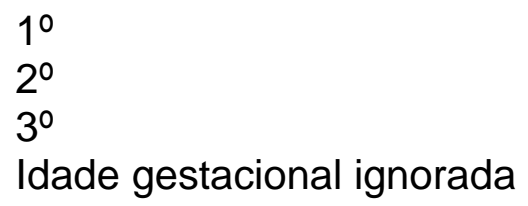

$\begin{array}{rr}1.271 & 48,9 \\ 720 & 27,7 \\ 569 & 21,9 \\ 39 & 1,5\end{array}$

\section{Escolaridade da gestante}

\begin{tabular}{|c|c|c|}
\hline Analfabeto & 7 & 0,3 \\
\hline $1^{\text {a }}$ a $4^{a}$ série incompleta & 134 & 5,1 \\
\hline $4^{\mathrm{a}}$ série completa & 86 & 3 , \\
\hline $5^{\underline{a}}$ a $8^{a}$ série incompleta & 567 & 21,8 \\
\hline Fundamental completo & 296 & 11,4 \\
\hline Médio incompleto & 408 & 15, \\
\hline Médio completo & 429 & 16 , \\
\hline Superior incompleto & 63 & 2, \\
\hline Superior completo & 33 & 1 , \\
\hline Ignorado & 576 & 22 \\
\hline
\end{tabular}

Fonte: Elaborado pelos autores (2021)

Tabela 2. Continuação das características sociodemográficas e epidemiológicas das gestantes com sífilis $(n=2.599)$, notificadas no SINAN no município de Curitiba, Paraná, 2014-2019.

\section{Variáveis da sífilis gestacional}

n

$\%$

\section{Raça ou cor da gestante}

Branca

Preta

Amarela

Parda

Indígena

Ignorada
2.073

111

51

291

2

71

2.069

9

197

4
79,8

4,3

2,0

11,1

0,1

2,7

\section{Esquema de tratamento**}

Penicilina

Outro esquema

Não realizado

Ignorado 
Do total de casos de sífilis congênita, $83,3 \%$ das gestantes realizaram o prénatal durante a gestação e $74,3 \%$ foram diagnosticadas durante o pré-natal. Entre as que realizaram o pré-natal, apenas $18,6 \%$ tiveram seus parceiros tratados. Já o tratamento da gestante foi inadequado em $63,7 \%$ dos casos, não realizado em $28,2 \%$, e adequado em $6,2 \%$ das gestantes. Entre essas mulheres, predomina a raça branca (78,3\%), a faixa etária de 20 a 29 anos $(54,3 \%)$ e a escolaridade ignorada ( $27,2 \%)$, seguida de mulheres com $5^{\mathrm{a}}$ a $8^{\mathrm{a}}$ série incompleta $(18,7 \%)$.

Com relação aos recém-nascidos (RN) diagnosticados com sífilis congênita, $86,2 \%$ tiveram o diagnóstico na fase recente da doença, não havendo nenhum caso identificado de sífilis congênita tardia. Sendo então esse o número total de casos classificados como vivos. E em $99 \%$ desse total, o diagnóstico foi feito em RN com menos de 7 dias de vida. Nenhum caso foi registrado de diagnóstico em crianças maiores de 2 anos nesse mesmo período. $13,8 \%$ dos casos totais de sífilis congênita notificados tiveram óbito pela doença, sendo $11,2 \%$ aborto por sífilis e $2,6 \%$ casos de natimortos (tabela 3) e (tabela 4).

Tabela 3. Características sociodemográficas e epidemiológicas da sífilis congênita (n=891), notificadas no SINAN no município de Curitiba, Paraná, 2014-2019.

\begin{tabular}{lrr}
\hline Variáveis da sífilis congênita & $\mathbf{n}$ & $\%$ \\
\hline Idade da criança no momento do diagnóstico & & \\
Menos de 7 dias & 882 & 99 \\
7 a 21 dias & 3 & 0,3 \\
28 a 364 dias & 5 & 0,6 \\
1 ano & 1 & 0,1 \\
2 a 4 anos & - & - \\
5 a 12 anos & - & - \\
Ignorado & - & - \\
Classificação clínica da doença no momento do diagnóstico & \\
Sífilis congênita recente & 768 & 86,2 \\
Sífilis congênita tardia & - & - \\
Aborto por sífilis & 100 & 11,2 \\
Natimorto por sífilis & 23 & 2,6 \\
\hline
\end{tabular}




\section{Faixa etária da mãe (em anos)}

10 a 14

15 a 19

20 a 29

484

54,3

30 a 39

219

40 ou mais

39

24,6

Ignorado

Escolaridade da mãe

\begin{tabular}{lrr} 
Analfabeto & 3 & 0,3 \\
$1^{\text {a }}$ a 4 $4^{\text {a }}$ série incompleta & 34 & 3,8 \\
$4^{\text {a }}$ série completa & 19 & 2,1 \\
$5^{\text {a }}$ a 8 8 série incompleta & 167 & 18,7 \\
Fundamental completo & 110 & 12,3 \\
Médio incompleto & 115 & 12,9 \\
Médio completo & 153 & 17,2 \\
Superior incompleto & 28 & 3,1 \\
Superior completo & 15 & 1,7 \\
Não se aplica & 5 & 0,6 \\
lgnorado & 242 & 27,2 \\
\hline
\end{tabular}

Fonte: Elaborado pelos autores (2021)

Tabela 4. Continuação das características sociodemográficas e epidemiológicas da sífilis congênita ( $n=891$ ), notificadas no SINAN no município de Curitiba, Paraná, 2014-2019

\begin{tabular}{lrr}
\hline Variáveis da sífilis congênita & $\mathbf{n}$ & $\%$ \\
\hline Raça ou cor da mãe & & \\
Branca & 698 & 78,3 \\
Preta & 27 & 3,0 \\
Amarela & 3 & 0,3 \\
Parda & 143 & 16 \\
Indígena & - & - \\
Ignorada & 20 & 2,2
\end{tabular}

\section{Realização do pré-natal pela mãe}

Sim

Não

\section{Momento do diagnóstico da sífilis materna}

Durante o pré-natal

No momento do parto/curetagem

Após o parto

Não realizado

Ignorado
662

193

33

3
74,3

21,7

3,7

0,3 


\section{Esquema de tratamento materno}

Adequado

Inadequado

Não realizado

Ignorado

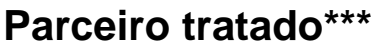

Sim

*** Dados de 2019 sobre o tratamento do parceiro não estão disponíveis. Fonte: Elaborado pelos autores (2021)

\section{DISCUSSÃO}

Este estudo demonstra que houve um aumento na prevalência da sífilis gestacional e na incidência da sífilis congênita durante o período de 2014 a 2019 na cidade de Curitiba. Esse acréscimo no número de casos, além de demonstrar uma taxa de transmissão ativa da doença, pode estar relacionado a um aumento no número das notificações e a um maior número de casos diagnosticados ${ }^{12}$, o que também foi notado em outras cidades brasileiras, como Palmas, Fortaleza e São Paulo ${ }^{4,12-14}$. Um dos fatores que pode ser responsável pelo acréscimo do número de notificação de casos foi um incremento dos serviços de saúde pública desenvolvidos durante o período do pré-natal ${ }^{12}$.

Em Curitiba, a Rede Mãe Curitibana Vale a Vida, antigo Programa Mãe Curitibana ${ }^{7}$, criado em 1999 pela Secretária Municipal de Saúde com o objetivo de reduzir a morbimortalidade materna e infantil, garante à gestante o acesso ao prénatal e realização de exames complementares, incluindo a realização de testagem para sífilis nos três trimestres de gestação ${ }^{15}$. Além disso, em 2009, foi implantado o Programa Pai Presente, que garante o acompanhamento e testagem para HIV e sífilis dos parceiros das gestantes do Programa Mãe Curitibana ${ }^{16}$. Uma das formas de rastreio da doença nas gestantes é a testagem durante o pré-natal (primeiro e terceiro trimestre), além da obrigatoriedade de realizar um teste treponêmico e um não treponêmico na maternidade ${ }^{3}$. Esses programas buscam garantir um pré-natal adequado, que inclui o atendimento da gestante em até 120 dias do início da gestação, 
realização de no mínimo seis consultas e oferta de exames laboratoriais básicos, como o teste rápido para sífilis e HIV ${ }^{12}$. Embora ocorram diversas possibilidades de identificação da doença durante a gravidez da mulher, o que possibilitaria uma redução da incidência de sífilis congênita, notou-se aumento maior que 50\% na incidência de sífilis congênita no ano de 2017 se comparado a 2014.

Pouco mais de 5\% das gestantes obtiveram o tratamento adequado em 2017, penicilina até 30 dias do parto, sendo a penicilina $G$ benzatina a medicação preconizada pelo Ministério da Saúde; e a dose terapêutica conforme o estágio da doença (primário, secundário ou latente). Fato que gera preocupação, visto que é estimado que $25 \%$ dos casos de sífilis gestacionais não tratadas resultem em óbito feta ${ }^{17}$. Além disso, é imprescindível o tratamento dos parceiros sexuais, pois esses são foco de infecção. Entretanto, como observado nesse estudo, apenas uma pequena parcela é tratada, perpetuando o número de casos e sendo um dos principais motivos de falha no tratamento integral da doença ${ }^{5,18}$.

A transmissão vertical da sífilis pode ocorrer em qualquer fase gestacional, mas é mais comum após o quarto mês ${ }^{5}$. Ademais, as taxas de transmissão estão diretamente relacionadas à carga treponêmica materna. Em decorrência disso, nas fases primárias e secundárias da doença a probabilidade de contaminação do feto pode chegar a $100 \%$, enquanto na fase latente precoce a transmissão é de $30 \%^{19}$. As consequências da sífilis para o recém-nato dependem da fase da infecção materna e da idade gestacional de quando ocorreu a exposição fetal ${ }^{20}$. A maioria dos bebês foi diagnosticada com sífilis congênita recente e com menos de sete dias, com alterações que podem ser interrompidas com o tratamento adequado ${ }^{19}$.

O perfil sociodemográfico das gestantes demonstrado aponta dados em conformidade com estudos realizados em outras cidades brasileiras quanto a faixa etária. No entanto, a cor da pele e classificação clínica variou quanto a localidade da cidade avaliada ${ }^{13-14}$. Em nosso estudo, nota-se a predominância de gestantes brancas com $79,8 \%$ e a sífilis latente com $88,9 \%$ dos casos. Isso pode indicar que a doença afeta um grupo heterogêneo de pessoas e, por isso, embora seja de fundamental importância direcionar a atenção das políticas públicas para a parte da população que seja mais afetada, é essencial realizar a promoção e prevenção de saúde à população como um todo ${ }^{21}$. 
Embora a cobertura de pré-natal no Brasil seja de $90 \%{ }^{13}$, a avaliação dos resultados demonstra a magnitude do problema da sífilis congênita. Pode-se inferir que a incidência de sífilis congênita em Curitiba ainda é superior ao estipulado ( 0,5 casos por 1000 nascidos vivos) pela Estratégia e Plano de Eliminação da Transmissão Vertical do HIV e da Sífilis Congênita da OPAS ${ }^{22}$, sendo esse valor também excedente à média estadual no mesmo período (2014-2019). Quando comparado com as demais capitais da região Sul, Curitiba é a que apresenta a menor taxa de incidência em todos os anos, variando de 5,5 (2014) a 8,3 (2017). Florianópolis possui dados com variação de incidência intermediária, e Porto Alegre é a capital com os indicadores mais expressivos entre as capitais da região Sul, com valores variando de 21,7 (2014) a $28,3(2018)^{23}$. Sem notificação adequada não há investigação nem tratamento, portanto é necessário investir em vigilância epidemiológica para controle da doença ${ }^{20}$.

\section{CONCLUSÃo}

Observa-se que o perfil das mulheres notificadas com sífilis gestacional em Curitiba possui em sua maioria entre 20 e 29 anos, são diagnosticadas no primeiro trimestre e em fase de sífilis latente. Em relação a sífilis congênita, a maioria dos casos notificados tiveram seu diagnóstico em fase de sífilis congênita recente, em mães que realizaram pré-natal e tratamento de forma inadequada. Nesse sentido, embora seja bem estabelecida a importância da realização de acompanhamentos pré-natais e do rastreio da sífilis nas gestantes com o objetivo de reduzir a sífilis gestacional e as consequências da sífilis congênita, nota-se que a capital paranaense ainda possui incidência de sífilis congênita superior a meta de 0,5 caso por 1.000 nascidos vivos proposta pela OPAS. Apesar disso, Curitiba é a capital do sul do Brasil com os menores indicadores de sífilis gestacional e congênita.

Por fim, o uso de dados secundários, a impossibilidade de estimar a frequência das subnotificações e a análise de forma observacional foram consideradas limitações de nossa pesquisa. Nesse sentido, reforçamos a necessidade de estudos que permitam quantificar a frequência das subnotificações para uma maior elucidação de nossos achados. 


\section{REFERÊNCIAS}

1. De Lorenzi DRS, Madi JM. Sífilis Congênita como Indicador de Assistência Prénatal. Rev. bras ginecol obstet. $2001 ; 23(10): 647-52$. [acesso em 2020 nov 21] doi: https://doi.org/10.1590/S0100-72032001001000006

2. World Health Organization - WHO. WHO Guideline for the treatment of Treponema pallidum (syphilis). Genebra: World Health Organization; 2016 [Acesso em: 2020 nov 6 ]. $60 \quad$ p. $\quad$ Disponível em: http://apps.who.int/iris/bitstream/10665/249572/1/9789241549806-eng.pdf

3. Ministério da Saúde (BR). Protocolo clinico e diretrizes terapêuticas para prevenção da transmissão vertical de HIV, Sífilis e Hepatites virais (2019). [Acesso em: 2021 jun 22] Disponível em: http://www.aids.gov.br/pt-br/pub/2015/protocoloclinico-e-diretrizes-terapeuticas-para-prevencao-da-transmissao-vertical-de-hiv

4. Cavalcante PAM, Pereira RBL, Castro JGD. Sífilis gestacional e congênita em Palmas, Tocantins, 2007-2014. Epidemiol. Serv. Saúde (Online). 2017;26(2):25564. [Acesso em: 2020 nov 11] doi: https://doi.org/10.5123/S1679$\underline{49742017000200003}$

5. Magalhães DMS, Kawaguchi IAL, Dias A, Calderon IMP. Sífilis materna e congênita: ainda um desafio. Cad. Saúde Pública (Online). 2013;29(6):1109-20. [Acesso em: 2021 jun 22] doi: https://doi.org/10.1590/S0102$\underline{311 \times 2013000600008}$

6. Sonda EC, Richter FF, Boschetti G, Casasola MP, Krumel CF, Machado CPH. Sífilis Congênita: uma revisão da literatura. Rev. epidemiol. controle infecç. 2013;3(1):28. [Acesso em 2021 jun 22] doi: http://dx.doi.org/10.17058/reci.v3i1.3022

7. Secretaria Municipal da Saúde de Curitiba. Rede Mãe Curitibana Vale a Vida Secretaria Municipal da Saúde. 2018 [Acesso em 2021 jun 22] Disponível em: https://www.fetalmed.net/wpcontent/uploads/2018/09/Protocolo Rede M\%C3\%A3e Curitiba Valea Vida web.pdf

8. Ministério da Saúde (Brasil). Sistema de Informação de Agravos de Notificação Sinan. [Internet]. Brasília: Ministério da Saúde, 2020. [Acesso em: 2021 jun 22]. Disponível em: http://indicadoressifilis.aids.gov.br

9. Instituto Brasileiro de Geografia e Estatística. Cidades@ [Internet]. Rio de Janeiro: Instituto Brasileiro de Geografia e Estatística; 2020. Disponível em: https://cidades.ibge.gov.br/brasil/pr/curitiba/panorama

10. Ministério da Saúde (Brasil). Secretaria de Vigilância em Saúde. Departamento de DST, Aids e Hepatites Virais. Bol. epidemiol. Sif. 2015;4(1):1-28. [Acesso em: 2021 jun 22] Disponível em: http://www.aids.gov.br/pt-br/node/88 
11. Ministério da Saúde (Brasil). Secretaria de Vigilância em Saúde. Programa Nacional de DST e Aids. Diretrizes para controle da Sífilis congênita. Brasília: Ministério da Saúde; 2005. 72p. [Acesso em: 2021 jun 22] Disponível em: https://bvsms.saude.gov.br/bvs/publicacoes/diretrizes controle sifilis congenita. pdf

12. Benito LAO, Souza WN. Perfil epidemiológico da sífilis congênita no Brasil no período de 2008 a 2014. Univ. Ciências da Saúde (Online). 2016;14(2). [Acesso em: 2021 jun 22] doi: https://doi.org/10.5102/ucs.v14i2.3811

13. Cardoso ARP, Araújo MAL, Cavalcante MS, Frota MA, Melo SP. Análise dos casos de sífilis gestacional e congênita nos anos de 2008 a 2010 em Fortaleza, Ceará, Brasil. Ciênc. Saúde Colet. 2018;23(2):563-74. [Acesso em: 2021 jun 22] doi: https://doi.org/10.1590/1413-81232018232.01772016

14. Maschio-Lima T, Machado ILL, Siqueira JPZ, Almeida MTG. Perfil epidemiológico de pacientes com sífilis congênita e gestacional em um município do Estado de São Paulo, Brasil. Rev. Bras. Saúde Matern. (Online) Infant. 2019;19(4):865-72. [Acesso em: 2021 jun 22] doi: https://doi.org/10.1590/1806-93042019000400007

15. Carvalho DS, Novaes HMD. Evaluation of the prenatal care program in Curitiba, Paraná, Brazil: a cohort study of primigravidae. Cad. Saúde Pública (Online).2004;20 Suppl2:220-30. [Acesso em: 2020 nov 20]. doi: https://doi.org/10.1590/1413-81232017223.18842016

16. Burger M. O Impacto do Programa Mãe Curitibana sobre a Transmissão Vertical do HIV no Município de Curitiba entre 2000 e 2009. DST j. bras. sex. transm. 2011;23(2):76-83. [Acesso em: 2021 jun 22] Disponível em: http://www.dst.uff.br/revista23-2-2011/60\%20Impacto\%20do\%20Programa\%20Mae\%20Curitibana.pdf

17. Domingues RMSM, Leal MC. [Incidence of congenital syphilis and factors associated with vertical transmission: data from the Birth in Brazil study]. Cad. Saúde Pública (Online). [Acesso em: 2021 jun 22] 2016; 32(6):1-12. doi: https://doi.org/10.1590/0102-311X00082415

18. Lafetá KRG, Martelli Júnior H, Silveira MF, Paranaíba LMR. Sífilis materna e congênita, subnotificação e difícil controle. Rer. bras. epidemiol. 2016;19(1):6374. [Acesso em: 2021 jun 22] doi: https://doi.org/10.1590/1980$\underline{5497201600010006}$

19. Sociedade Brasileira de Pediatria. Tratado de Pediatria. $4^{a}$ ed, vol 1. São Paulo: Manole; 2017. p 1008-1012.

20. Padovani C, Oliveira RR, Pelloso SM. Syphilis in during pregnancy: Association of maternal and perinatal characteristics in a region of southern Brazil. Rev. latinoam. enferm. (Online). 2018;26. [Acesso em: 2021 jun 22] doi: https://doi.org/10.1590/1518-8345.2305.3019

21. Bottura BR, Matuda L, Rodrigues PSS, Amaral CMCA, Barbosa LG. Perfil 
epidemiológico da sífilis gestacional e congênita no Brasil - período de 2007 a 2016. Arq. méd. hosp. Fac. Ciênc. Méd. Santa Casa São Paulo. 2019;64(2):69. [Acesso em: 2021 jun 22] doi: https://doi.org/10.26432/1809-3019.2019.64.2.069

22. Arnesen L, Serruya S, Durán P. Gestational syphilis and stillbirth in the Americas: A systematic review and meta-analysis. Ver. panam. salud pública. 2015;37(6):422-9. [Acesso em: 2021 jun 22] Disponível em: https://www.scielosp.org/article/rpsp/2015.v37n6/422-429/en/

23. Ministério da Saúde (Brasil). Departamento de Doenças de Condições Crônicas e Infecções Sexualmente Transmissíveis. Indicadores e dados básicos da sífilis nos municípios brasileiros. Brasília: Ministério da Saúde; 2020. [Acesso em 2020 nov 20] Disponível em: http://indicadoressifilis.aids.gov.br 